\title{
Land suitability classification of some Qalubiya soils
}

\author{
Abd El-Hameed, A.H. ${ }^{1}$, Naufal, E.H. ${ }^{1}$, El-Husseiny, O. H. ${ }^{1}$, Mohamed, M.K ${ }^{2}$ and Abuzaid , A. S. ${ }^{1}$ \\ Soils Department, Faculty of Agriculture, Benha University, Egypt ${ }^{1}$ \\ Soils, Water and Environment Research Institute, Agricultural Research Center, Giza. ${ }^{2}$
}

\begin{abstract}
The present work aimed at sassing land suitability for irrigated agriculture and certain crops of some soils in Qalubiya Governorate, Egypt. The studied area covers an arable area of about $81000 \mathrm{ha}\left(810 \mathrm{~km}^{2}\right)$, located on Damietta branch, east of the Nile River, between latitudes $30^{\circ} 06^{\prime}$ and $30^{\circ} 36^{\prime} \mathrm{N}$ and longitudes $31^{\circ} 03^{\prime}$ to $31^{\circ}$ $35^{\prime} \mathrm{E}$. Twenty soil profiles representing the main physiographic units have been chosen to represent the studied area. The soils are of S1 class (highly suitable), S2 (moderately suitable) and S3 (marginally suitable). Currently, the soils are related to three sub classes, i.e. S2s: with slight intensity of texture, gypsum and salinity and alkalinity limitations; S2sn: with moderate intensity of salinity and alkalinity and slight intensity of texture limitations and S3s: with severe intensity of texture limitation. Potentially, the soils are classified into two subclasses, i.e. S2s: with slight (S2s-1) and severe (S2s-2) intensity of texture limitations and S3s: with severe intensity of texture limitations. The soils are suitable in their current conditions for most of selected crops, although some soils appears not suitable (N1) for cotton, carrots, onion, tomatoes, banana, guava and mango. By performing some improvements on the studied soils, they would be suitable in their potential conditions for all the selected crops.
\end{abstract}

Key words: Land suitability, irrigated agriculture, certain crops, Qalubiya soils.

\section{Introduction}

Land suitability is the fitness of a given type of land for a defined use. The process of land suitability classification is the appraisal and grouping of specific areas of land in terms of their suitability for defined uses (FAO, 1976). Actual land suitability is based on current soil and land conditions without applying any input. Potential land suitability is the suitability that could be reached after the land is improved (Ritung et al. 2007). Crop growth and yield are determined by a number of factors such as genetic potential for crop cultivation, soil, weather, cultivation practice and biotic stress (Singh Alka et al., 2008). Each plant species requires definite soil and site conditions for its optimum growth. Although some plants may grow on different soils and extreme agro-ecological conditions, not all plants can grow on one particular soil and under the same environment (Mishra, 2007). Sys (1985) and Sys et al. (1993) presented an approach which can be applied in various ecological types depending upon making a comparison between land characteristics or qualities and crop requirements.

Qalubiya Governorate is located on the Damietta branch, east of the Nile River, near the Delta head between latitudes $30^{\circ} 06^{\prime}$ and $30^{\circ} 36^{\prime} \mathrm{N}$ and longitudes $31^{\circ} 03^{\prime}$ to $31^{\circ} 35^{\prime} \mathrm{E}$, covering a total area of $1001 \mathrm{~km}^{2}$ and a cultivated area is $810 \mathrm{~km}^{2}$ (Shalaby and Gad, 2010). The elevation is $15 \mathrm{~m}$ a.s.l. from south to north and between 15 and $50 \mathrm{~m}$ a.s.l. from east to west (El-Shanawany, 1992). The present work aims at assessing land suitability for irrigated agriculture of some soils in the area in terms of land suitability for growing certain crops.

\section{Materials and methods}

\section{The field work}

Twenty soil profile pits were dug in the studied area (Table, 1 and Fig. 1). They were morphologically descried according to the methods outlined by FAO (2006) and Soil Survey Staff (2009). Soil color was described according to Munsell Color Charts (1971). Sixty soil samples were collected for different analyses.

\section{Laboratory work}

Samples were air dried, crushed and passed throw a $2 \mathrm{~mm}$ sieve. Chemical and physical analyses of were performed according to the standard methods outlined by USDA (2009).

3. Land suitability classification

It was done using the developed system of Sys and Verheye (1978), modified by Sys et al. (1991), concerning the FAO Framework for Land Evaluation (FAO, 1976). Further land suitability for certain crops was performed using the system suggested by Sys et al. (1993). The formula used to calculate land index is: $\mathrm{Si}=\mathrm{t} \times \frac{\mathrm{w}}{100} \times \frac{\mathrm{s}_{1}}{100} \times \frac{\mathrm{s}_{2}}{100} \times \frac{\mathrm{s}_{3}}{100} \times \frac{\mathrm{s}_{4}}{100} \times \frac{\mathrm{n}}{100}$

Where: $\mathrm{Si}=$ suitability index; $\mathrm{t}=$ slope; $\mathrm{w}=$ drainage; $s_{1}=$ texture; $s_{2}=$ depth; $s_{3}=\mathrm{CaCO}_{3}$ content; $\mathrm{s}_{4}=$ gypsum content and $\mathrm{n}=$ salinity and alkalinity (sodicity).

Suitability is arbitrarily defined according to the value of the suitability index as shown in Table 2 . The limitation scale can be completely done by a parametric method of evaluation. The ratings to be selected for different limitation levels are present in Table 3. 
Table 1. Distribution of the geomorphic units in the studied area of Qalubiya Governorate. Geomorphic unit Profile number Location

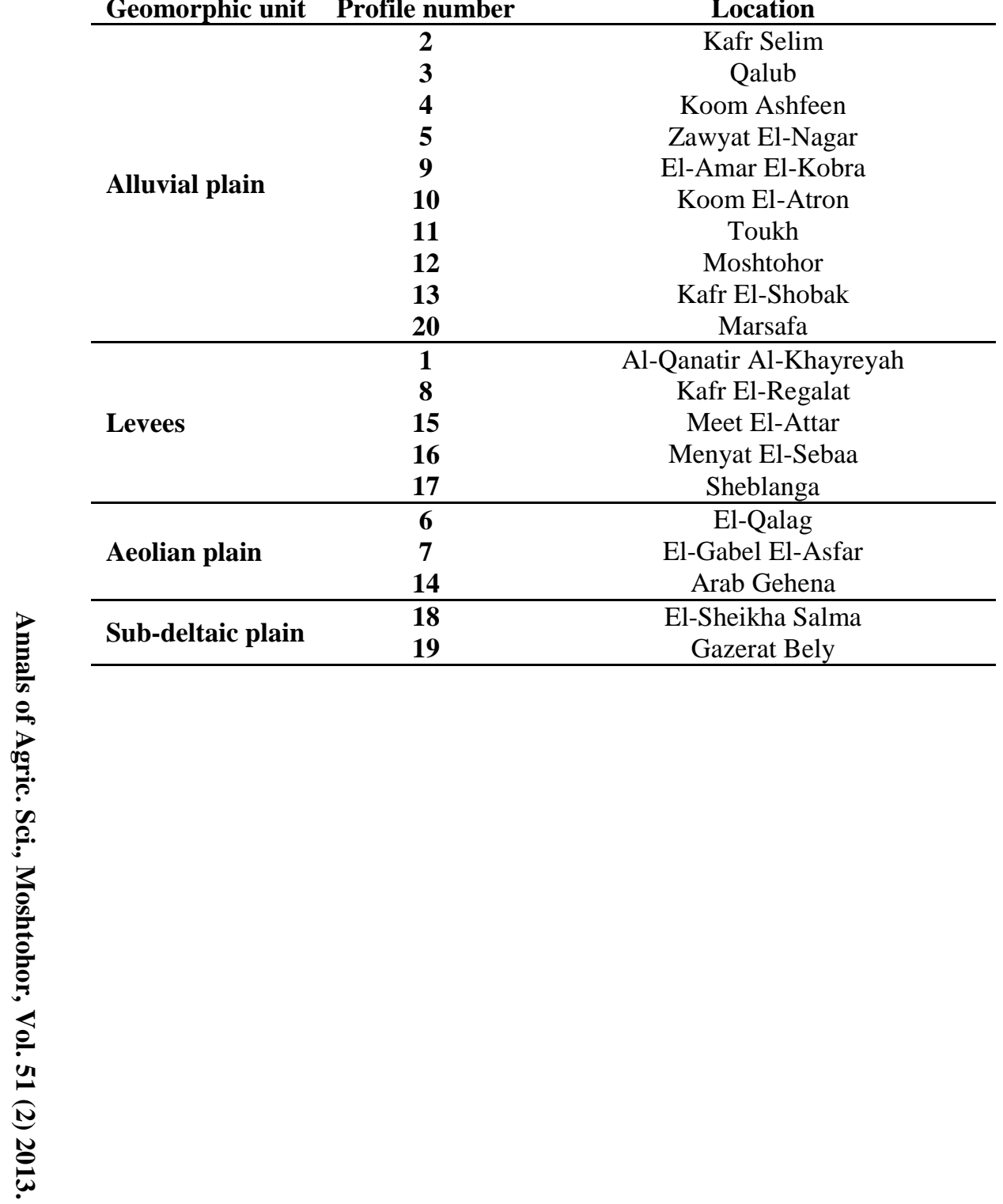

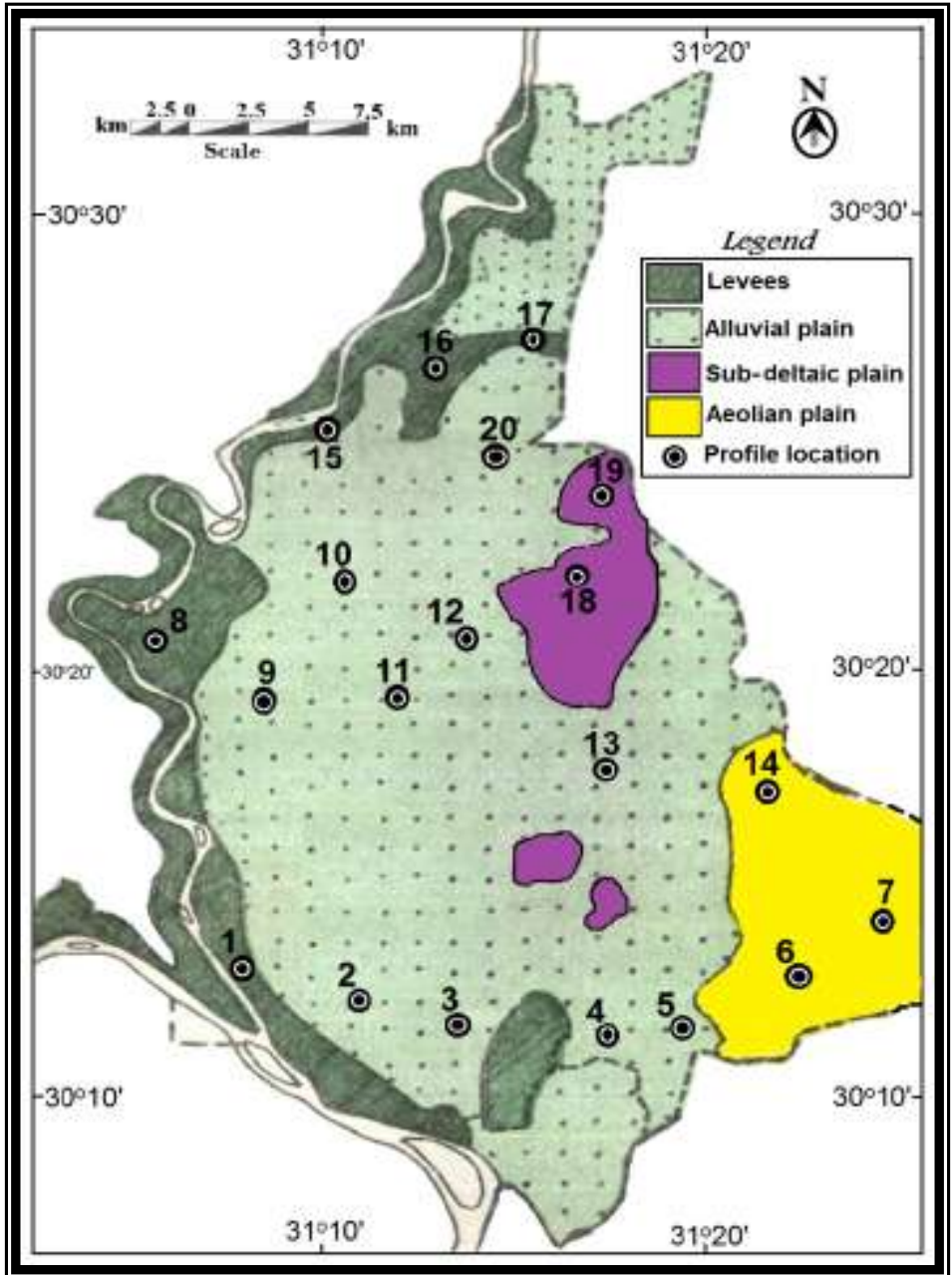

Fig. 1. Distribution of soil profiles representing the studied area of Qalubiya Governorate 
Table 2. Land suitability categories.

\begin{tabular}{|c|c|c|c|c|}
\hline Order & Symbol & Class & Symbol & Suitability index (Si) \\
\hline \multirow{3}{*}{ Suitable } & \multirow{3}{*}{$\mathrm{S}$} & Highly suitable & S1 & $>75$ \\
\hline & & Moderately suitable & $\mathrm{S} 2$ & $75-50$ \\
\hline & & Marginally suitable & S3 & $50-25$ \\
\hline \multirow{2}{*}{ Not suitable } & \multirow{2}{*}{$\mathrm{N}$} & Currently not suitable & N1 & $<25$ \\
\hline & & Permanently not suitable & $\mathrm{N} 2$ & $<25$ \\
\hline \multicolumn{5}{|c|}{ Table 3. Limitation levels and their ratings } \\
\hline Symbol & & Intensity of limita & & Rating \\
\hline 0 & & No & & $100-95$ \\
\hline 1 & & Slight & & $95-85$ \\
\hline 2 & & Moderate & & $85-60$ \\
\hline 3 & & Severe & & $60-40$ \\
\hline 4 & & Very Severe & & $40-0$ \\
\hline
\end{tabular}

\section{Results and discussions}

\section{Soil characteristics (Table 4)}

\section{Soil reaction $(\mathbf{p H})$}

The soils have neutral to strongly alkaline reaction (Soil Survey Staff, 1993) as the $\mathrm{pH}$ values varied from 6.65 to 8.77 . The relatively low $\mathrm{pH}$ value, characterized the surface horizon of soil profile No. 7 in El-Gabal El-Asfar farm, is probably due to the usage of sewage water for irrigation for several years. This water is enriched with organic residues that would accumulate on soil surface. Decomposition of such residues produces organic acids that decrease soil reaction (Abd El-Salam, 2001 and Bassuony, 2012).

\section{Soil salinity}

The soils are none saline to very slightly saline (Soil Survey Staff, 1993) since the EC values range from 0.17 to $3.45 \mathrm{dSm}^{-1}$. This may be due to: 1$)$ the native rocks from which the soils originated, have minor amounts of soluble salts; 2) existence of drainage systems allowing the removal of excess salts; and/or 3) the usage of the Nile water which is a high quality water for irrigation. On the other hand, the high values of EC observed in some profiles, may be due to the usage of low quality water for irrigation including ground water and sewage water (in ElGabel El-Asfar) where Nile water is not available.

\section{Exchange characteristics}

Values of the CEC fluctuated between 3.91 and $51.13 \mathrm{cmol}_{\mathrm{C} g}{ }^{-1}$ soil. ESP ranged between 2.29 and 18.79. The wide variation of CEC seemed to be dependant on both the clay and the organic matter contents where the soils with high contents of the fine fractions components achieves high CEC values and in contrast, soils with high content of coarse fraction have small specific surface area and consequently, low CEC values.

\section{Organic matter}

It ranged from low to adequate (Ryan et al., 2003)), since it varied from 1.60 to $25.30 \mathrm{gkg}^{-1}$. These relatively high values may be attributed application of bio and organic fertilizers that enriched soils with organic carbon. Rice (2002), Bot and Benites (2005) and Samy and Metwally (2012) showed that soil organic matter tends to increase as the clay content increases. They attributed this to bonds between surface of clay particles and organic matter retarding decomposition of organic matter and increase the potential for aggregate formation that protects organic matter from further mineralization. On the other hand, the low values of organic matter in some profiles may be a result of aridity that accelerates oxidation and decomposition of soil organic matter as well as to dominance of coarse fraction.

\section{Calcium carbonate content}

Calcium carbonate ranged from 6.70 and 29.30 $\mathrm{gkg}^{-1}$. This may be because the parent materials from which the soils were derived had low amount of calcite and/or entire absence of carbonate ions in both soil solution and irrigation water (Brewer, 1964; Ryan et al., 2003 and Bassuony, 2012).

\section{Gypsum content}

Gypsum ranged from 7.70 to $29.20 \mathrm{gkg}^{-1}$ because one or more of the following reasons: in situ weathering of existing parent material did not reach its optimal level to accumulate gypsum, low sulfateenriched precipitations, low accumulation of aeolian or fluvial sulfate-rich sediment, or slow rate of in situ oxidation of sulfide minerals (Buck and Van Hoesen, 2002 and Hashemi et al., 2011).

\section{Soil texture}

Five textural classes are identified in the studied area, i.e. clay, clay loam, sandy clay loam, loamy sand and sand. All the studied soil profiles almost showed a pattern of homogeneity in textural classes within different horizons of the same profile except for the soil profiles No. 14 and 18 that involved two textural classes, loamy sand and sand. 
Table 4. Soil characteristics of the studied geomorphic units.

\begin{tabular}{|c|c|c|c|c|c|c|c|c|c|c|}
\hline Geomorphic unit & $\begin{array}{c}\text { Profile } \\
\text { No. }\end{array}$ & $\begin{array}{l}\text { Depth } \\
\text { (cm) }\end{array}$ & pH & $\begin{array}{c}\mathrm{EC} \\
\left(\mathrm{dSm}^{-1}\right)\end{array}$ & $\begin{array}{c}\text { CEC } \\
\left(\mathrm{cmol}_{\mathrm{C}} \mathbf{k g}^{-1}\right)\end{array}$ & ESP & $\underset{\left(\mathrm{gkg}^{-1}\right)}{\mathrm{OM}}$ & $\begin{array}{c}\mathrm{CaCO}_{3} \\
\left(\mathrm{gkg}^{-1}\right)\end{array}$ & $\begin{array}{c}\text { Gypsum } \\
\left(\mathrm{gkg}^{-1}\right)\end{array}$ & Textural class \\
\hline \multirow{30}{*}{ Alluvial plain } & \multirow{4}{*}{2} & $0-30$ & 7.79 & 1.16 & 50.43 & 8.95 & 18.20 & 22.40 & 12.50 & Clay \\
\hline & & $30-60$ & 7.48 & 1.21 & 51.13 & 9.13 & 16.00 & 20.40 & 15.10 & Clay \\
\hline & & $60-150$ & 7.80 & 1.16 & 50.43 & 7.88 & 16.60 & 20.00 & 14.30 & Clay \\
\hline & & $0-35$ & 7.09 & 1.12 & 46.52 & 11.48 & 24.80 & 22.00 & 12.50 & Clay \\
\hline & \multirow[t]{2}{*}{3} & $35-80$ & 7.47 & 0.50 & 46.09 & 9.16 & 19.00 & 21.30 & 13.60 & Clay \\
\hline & & $80-150$ & 7.59 & 0.45 & 45.22 & 10.29 & 17.10 & 21.00 & 8.40 & Clay \\
\hline & \multirow{3}{*}{4} & $0-40$ & 7.47 & 2.04 & 46.96 & 9.25 & 23.40 & 23.00 & 12.40 & Clay \\
\hline & & $40-65$ & 7.70 & 3.40 & 46.96 & 9.57 & 18.10 & 21.70 & 12.00 & Clay \\
\hline & & $65-150$ & 7.78 & 3.35 & 46.96 & 9.68 & 15.50 & 21.00 & 12.20 & Clay \\
\hline & \multirow{3}{*}{5} & $0-25$ & 7.87 & 0.82 & 44.35 & 7.03 & 19.10 & 28.80 & 22.70 & Clay \\
\hline & & $25-60$ & 7.69 & 1.77 & 45.22 & 8.19 & 14.50 & 28.70 & 29.20 & Clay \\
\hline & & $60-150$ & 7.86 & 0.59 & 45.65 & 7.31 & 11.10 & 29.30 & 13.40 & Clay \\
\hline & \multirow{3}{*}{9} & $0-25$ & 7.88 & 0.45 & 43.48 & 9.90 & 23.80 & 22.00 & 8.40 & Clay \\
\hline & & $25-55$ & 8.13 & 0.58 & 44.35 & 13.97 & 24.30 & 19.50 & 10.10 & Clay \\
\hline & & $55-150$ & 8.25 & 0.76 & 43.13 & 18.79 & 18.10 & 21.40 & 11.80 & Clay \\
\hline & \multirow{4}{*}{10} & $0-30$ & 7.53 & 0.43 & 43.48 & 7.46 & 25.30 & 20.30 & 8.40 & Clay \\
\hline & & $30-60$ & 7.80 & 0.35 & 46.09 & 8.72 & 22.20 & 22.50 & 12.80 & Clay \\
\hline & & $60-150$ & 8.04 & 0.38 & 46.96 & 12.83 & 20.70 & 19.60 & 13.60 & Clay \\
\hline & & $0-50$ & 7.89 & 0.57 & 41.74 & 5.85 & 18.60 & 21.50 & 11.90 & Clay \\
\hline & \multirow[t]{2}{*}{11} & $50-80$ & 7.62 & 0.72 & 39.57 & 7.08 & 16.60 & 21.50 & 8.30 & Clay \\
\hline & & $80-150$ & 7.64 & 0.43 & 36.52 & 8.49 & 18.60 & 19.50 & 14.40 & Clay \\
\hline & \multirow{3}{*}{12} & $0-40$ & 7.72 & 0.57 & 40 & 7.33 & 13.40 & 21.40 & 8.30 & Clay \\
\hline & & $40-70$ & 7.88 & 0.74 & 39.57 & 7.08 & 18.20 & 18.70 & 9.30 & Clay \\
\hline & & $70-150$ & 7.73 & 1.86 & 39.13 & 7.08 & 24.80 & 18.00 & 9.80 & Clay \\
\hline & \multirow{3}{*}{13} & $0-40$ & 7.53 & 0.33 & 40.87 & 8.73 & 21.70 & 18.00 & 8.50 & Clay \\
\hline & & $40-65$ & 7.55 & 0.35 & 41.74 & 9.34 & 14.00 & 18.40 & 9.30 & Clay \\
\hline & & $65-150$ & 7.62 & 0.27 & 39.13 & 6.52 & 21.20 & 18.40 & 10.20 & Clay \\
\hline & \multirow{3}{*}{20} & $0-30$ & 7.42 & 0.53 & 45.22 & 7.8 & 24.50 & 21.50 & 11.80 & Clay \\
\hline & & $30-50$ & 7.74 & 0.36 & 44.35 & 5.66 & 17.80 & 22.00 & 10.20 & Clay \\
\hline & & $50-150$ & 7.81 & 0.43 & 43.48 & 6.66 & 18.60 & 22.00 & 11.90 & Clay \\
\hline
\end{tabular}

* Textural class according to the USDA Texture triangle 
Table 4. Cont.

\begin{tabular}{|c|c|c|c|c|c|c|c|c|c|c|}
\hline Geomorphic unit & Profile No. & Depth (cm) & $\mathbf{p H}$ & $\begin{array}{c}\mathrm{EC} \\
\left(\mathrm{dSm}^{-1}\right)\end{array}$ & $\begin{array}{c}\text { CEC } \\
\left(\mathrm{cmol}_{\mathbf{C}} \mathbf{k g}^{-1}\right)\end{array}$ & ESP & $\underset{\left(\mathrm{gkg}^{-1}\right)}{\mathrm{OM}}$ & $\begin{array}{c}\mathrm{CaCO}_{3} \\
\left(\mathrm{gkg}^{-1}\right)\end{array}$ & $\begin{array}{c}\text { Gypsum } \\
\left(\mathrm{gkg}^{-1}\right)\end{array}$ & Textural class \\
\hline \multirow{15}{*}{ Levees } & \multirow{3}{*}{1} & $0-25$ & 7.37 & 0.86 & 45.22 & 4.05 & 17.10 & 20.30 & 16.80 & Clay \\
\hline & & $26-60$ & 7.48 & 0.40 & 46.09 & 7.42 & 15.50 & 20.80 & 14.50 & Clay \\
\hline & & $60-150$ & 7.45 & 0.41 & 44.78 & 8.85 & 16.00 & 20.00 & 15.30 & Clay \\
\hline & \multirow{3}{*}{8} & $0-25$ & 7.44 & 0.40 & 28.7 & 6.78 & 12.40 & 18.00 & 9.30 & Clay loam \\
\hline & & $25-55$ & 7.55 & 0.17 & 24.78 & 7.07 & 10.30 & 17.00 & 12.80 & Clay loam \\
\hline & & $55-150$ & 7.64 & 0.27 & 25.65 & 8.46 & 15.50 & 19.00 & 13.60 & Clay loam \\
\hline & \multirow{3}{*}{15} & $0-40$ & 7.51 & 0.93 & 29.57 & 5.69 & 24.80 & 16.00 & 9.90 & Clay loam \\
\hline & & $40-65$ & 7.67 & 0.41 & 24.35 & 4.57 & 20.70 & 25.50 & 9.30 & Clay loam \\
\hline & & $65-150$ & 7.76 & 0.49 & 25.22 & 6.68 & 23.80 & 17.30 & 10.10 & Clay loam \\
\hline & \multirow{3}{*}{16} & $0-50$ & 7.47 & 0.67 & 43.48 & 7.79 & 24.10 & 18.60 & 10.00 & Clay \\
\hline & & $50-75$ & 7.74 & 0.37 & 45.22 & 7.59 & 20.70 & 17.60 & 17.10 & Clay \\
\hline & & $75-150$ & 7.75 & 0.39 & 44.35 & 2.29 & 22.80 & 17.20 & 15.30 & Clay \\
\hline & \multirow{3}{*}{17} & $0-35$ & 7.71 & 0.75 & 45.22 & 8.02 & 24.30 & 23.00 & 10.80 & Clay \\
\hline & & $35-60$ & 7.73 & 0.57 & 46.09 & 8.76 & 21.20 & 19.40 & 16.00 & Clay \\
\hline & & $60-150$ & 7.71 & 0.68 & 43.48 & 6.3 & 19.40 & 19.50 & 10.90 & Clay \\
\hline \multirow{8}{*}{ Aeolian plain } & \multirow{3}{*}{6} & $0-35$ & 7.61 & 0.79 & 26.96 & 4.75 & 25.00 & 19.00 & 12.70 & Sandy clay loam \\
\hline & & $35-60$ & 7.79 & 0.63 & 25.65 & 4.84 & 20.10 & 22.40 & 8.40 & Sandy clay loam \\
\hline & & $60-150$ & 7.82 & 0.56 & 24.35 & 5.07 & 10.30 & 23.00 & 13.50 & Sandy clay loam \\
\hline & \multirow{3}{*}{7} & $0-50$ & 6.65 & 3.45 & 8.7 & 3.87 & 10.90 & 10.20 & 19.80 & Loamy sand \\
\hline & & $50-90$ & 6.96 & 0.38 & 4.78 & 2.96 & 10.60 & 6.70 & 13.70 & Loamy sand \\
\hline & & $90-150$ & 7.71 & 0.78 & 5.22 & 5.54 & 9.80 & 6.80 & 21.40 & Loamy sand \\
\hline & \multirow{2}{*}{14} & $0-45$ & 7.35 & 2.56 & 7.83 & 6.75 & 7.20 & 15.00 & 12.30 & Loamy sand \\
\hline & & $45-65$ & 7.71 & 2.59 & 5.22 & 10.16 & 5.20 & 11.80 & 12.30 & Loamy sand \\
\hline \multirow{7}{*}{ Sub-deltaic plain } & \multirow{5}{*}{18} & $65-150$ & 8.04 & 2.10 & 4.96 & 11.54 & 5.70 & 12.20 & 12.60 & Sand \\
\hline & & $0-50$ & 7.78 & 0.53 & 7.83 & 5.97 & 3.10 & 11.20 & 8.50 & Loamy sand \\
\hline & & $50-80$ & 7.85 & 0.36 & 5.22 & 5.25 & 2.10 & 15.40 & 13.70 & Loamy sand \\
\hline & & $80-150$ & 8.34 & 0.32 & 4.35 & 17.55 & 1.60 & 13.40 & 10.30 & Loamy sand \\
\hline & & $0-50$ & 7.61 & 0.75 & 8.7 & 7.06 & 4.70 & 21.70 & 8.50 & Loamy sand \\
\hline & \multirow[t]{2}{*}{19} & $50-90$ & 7.50 & 0.46 & 5.22 & 5.54 & 3.60 & 12.00 & 7.70 & Loamy sand \\
\hline & & $90-150$ & 8.77 & 0.30 & 3.91 & 18.35 & 3.30 & 11.50 & 7.70 & Sand \\
\hline
\end{tabular}




\section{Land suitability classification}

\section{Current suitability}

Current suitability classification may refer to the present use of the land, either with existing or improved management practices, or to a different use (FAO, 1976). Results in Table 5 indicate that values of suitability index ( $\mathrm{Si}$ ) is more than 25, which means that soils are suitable (S) for irrigated agriculture. These values ranged from 41 to 91 . The soils could be classified into three classes, i.e. highly suitable (S1), moderately suitable (S2) and marginally suitable (S3). Three subclasses were identified, i.e. S2s: moderately suitable with slight intensity of texture, gypsum, salinity and alkalinity limitations; S2sn: moderately suitable with moderate salinity and alkalinity and slight intensity of texture limitations and S3s: marginally suitable with severe intensity of texture limitation.

\section{Potential suitability}

Potential suitability refers to the suitability, for a defined use, of land units in their condition at some future date, after specified major improvements have been completed. For a classification to be done for potential suitability, not all improvements must be made to all of the land and the need for major improvements may vary from one land unit to another (FAO, 1976).

Land leveling for adjusting slope and application of calcium bearing compounds (such as gypsum) and leaching of excessive salts to reduce salinity and alkalinity hazards are the most vital land improvements in the studied area. Application of organic fertilizers and green manures and constructing modern irrigation systems as drip or sprinkler irrigation are of a great importance for increasing suitability of coarse textured soils.

Potential suitability could be classified into the order suitable (S), three classes (S1, S2 and S3) and two subclasses (S2s and S3s). The subclass S2s, moderately suitable with texture limitations is distributed into two units, i.e. S2s-1 with slight intensity of texture and gypsum limitations and S2s-2 with sever intensity of texture limitation. The subclass S3s, marginally suitable, has severe intensity of texture limitation.

\section{Land suitability classification for certain crops}

Twelve crops were selected to asse their convenience for cultivation in the studied area. The selected crops could be grouped into three categories as follows:
1. Field crops: cotton, groundnut, maize and wheat

2. Vegetable crops: cabbage, carrots, onion and tomato

3. Fruit crops: banana, citrus, guava and mango.

\section{Current land suitability}

Results in Table 6 indicate that nearly all studied soils are suitable (S1, S2 and S3) for growing all selected crops. However, some soils are not suitable (N1) for some selected crops. Regarding field crops, soils represented by profiles No. 14, 18 and 19 are not suitable for cotton. Concerning vegetable crops, soils represented by profiles No. 2, 4, 5, 9, 11, 12, 1 , 17, 14, 18 are not suitable for carrots. Soils represented by profiles No. 7 and 14 are not suitable for onion. Soils represented by profile No. 18 are not suitable for tomato. Regarding fruit crops, nearly all the studied soils are not suitable (N1) for banana. Some soils appear marginally suitable (S3) among these soils those represented by profiles No. 10, 13, 20 and all the studied soils in levees unit. Soils represented by profiles No. 7, 14, 18 and 19 are not suitable (N1) for guava and mango.

\section{Potential suitability}

By applying the aforementioned land improvements, all soils in the area of study would be suitable for all the selected crops. The percentages of each suitability class for different crops in the studied area are in Table 7. From the economic point of view, cultivation of only highly suitable (S1) and moderately suitable (S2) crops is recommended. 
Table 5. Rating of limitations and land suitability of the geomorphic units.

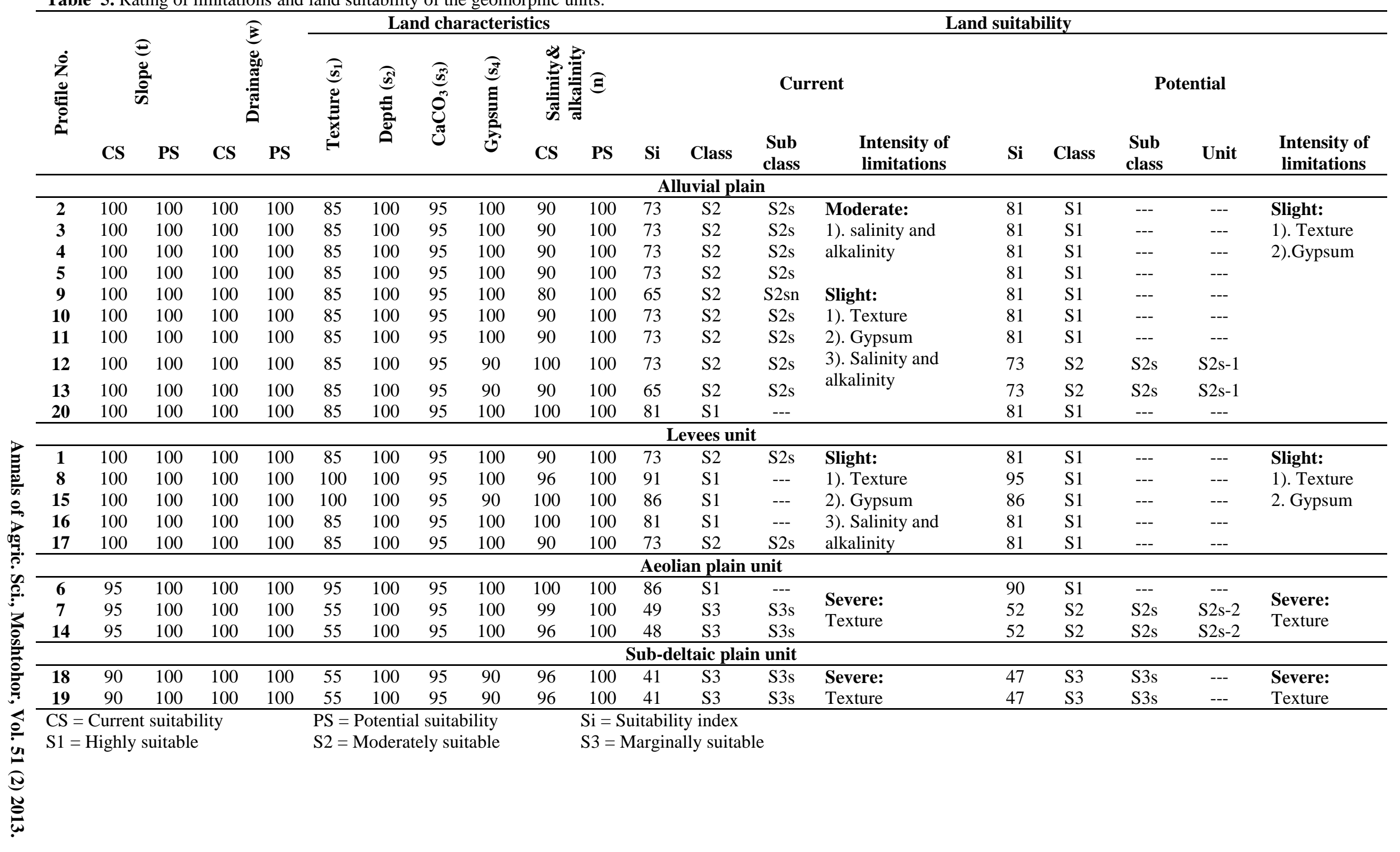


Table 6. Land suitability for the selected crops

\begin{tabular}{|c|c|c|c|c|c|c|c|c|c|c|c|c|c|c|c|c|c|c|c|c|c|c|c|c|}
\hline \multirow{3}{*}{$\begin{array}{c}\text { Profile } \\
\text { No. }\end{array}$} & \multicolumn{8}{|c|}{ Filed crops } & \multicolumn{8}{|c|}{ Vegetable crops } & \multicolumn{8}{|c|}{ Fruit crops } \\
\hline & \multicolumn{2}{|c|}{ Cotton } & \multicolumn{2}{|c|}{ Groundnuts } & \multicolumn{2}{|c|}{ Maize } & \multicolumn{2}{|c|}{ Wheat } & \multicolumn{2}{|c|}{ Cabbage } & \multicolumn{2}{|c|}{ Carrots } & \multicolumn{2}{|c|}{ Onion } & \multicolumn{2}{|c|}{ Tomato } & \multicolumn{2}{|c|}{ Bananas } & \multicolumn{2}{|c|}{ Citrus } & \multicolumn{2}{|c|}{ Guava } & \multicolumn{2}{|c|}{ Mango } \\
\hline & $\mathrm{CS}$ & PS & $\mathrm{CS}$ & PS & $\mathrm{CS}$ & PS & $\mathrm{CS}$ & PS & $\mathrm{CS}$ & PS & CS & PS & $\mathrm{CS}$ & PS & $\mathrm{CS}$ & PS & $\mathrm{CS}$ & PS & $\mathrm{CS}$ & PS & $\mathrm{CS}$ & PS & $\mathrm{CS}$ & PS \\
\hline \multicolumn{25}{|c|}{ Alluvial plain unit } \\
\hline 2 & $\begin{array}{c}52 \\
(\mathrm{~S} 2)\end{array}$ & $\begin{array}{c}97 \\
(\mathrm{~S} 1)\end{array}$ & $\begin{array}{c}35 \\
\text { (S3) }\end{array}$ & $\begin{array}{c}62 \\
(\mathrm{~S} 2)\end{array}$ & $\begin{array}{c}54 \\
(\mathrm{~S} 2)\end{array}$ & $\begin{array}{c}91 \\
(\mathrm{~S} 1)\end{array}$ & $\begin{array}{c}70 \\
\text { (S2) }\end{array}$ & $\begin{array}{c}98 \\
(\mathrm{~S} 1)\end{array}$ & $\begin{array}{c}59 \\
\text { (S2) }\end{array}$ & $\begin{array}{c}85 \\
(\mathrm{~S} 1)\end{array}$ & $\begin{array}{c}18 \\
\text { (N1) }\end{array}$ & $\begin{array}{c}53 \\
\text { (S2) }\end{array}$ & $\begin{array}{c}43 \\
\text { (S3) }\end{array}$ & $\begin{array}{c}70 \\
(\mathrm{~S} 2)\end{array}$ & $\begin{array}{c}41 \\
\text { (S3) }\end{array}$ & $\begin{array}{c}75 \\
(\mathrm{~S} 1)\end{array}$ & $\begin{array}{c}19 \\
\text { (N1) }\end{array}$ & $\begin{array}{c}74 \\
(\mathrm{~S} 2)\end{array}$ & $\begin{array}{c}37 \\
\text { (S3) }\end{array}$ & $\begin{array}{c}75 \\
(\mathrm{~S} 1)\end{array}$ & $\begin{array}{c}54 \\
(\mathrm{~S} 2)\end{array}$ & $\begin{array}{c}98 \\
(\mathrm{~S} 1)\end{array}$ & $\begin{array}{c}44 \\
\text { (S3) }\end{array}$ & $\begin{array}{c}75 \\
\text { (S1) }\end{array}$ \\
\hline 3 & $\begin{array}{c}87 \\
(\mathrm{~S} 1)\end{array}$ & $\begin{array}{c}97 \\
\text { (S1) }\end{array}$ & $\begin{array}{c}44 \\
(\mathrm{~S} 3)\end{array}$ & $\begin{array}{c}63 \\
(\mathrm{~S} 2)\end{array}$ & $\begin{array}{c}76 \\
\text { (S1) }\end{array}$ & $\begin{array}{c}92 \\
\text { (S1) }\end{array}$ & $\begin{array}{c}84 \\
(\mathrm{~S} 1)\end{array}$ & $\begin{array}{c}98 \\
(\mathrm{~S} 1)\end{array}$ & $\begin{array}{c}70 \\
\text { (S2) }\end{array}$ & $\begin{array}{c}86 \\
(\mathrm{~S} 1)\end{array}$ & $\begin{array}{c}35 \\
\text { (S3) }\end{array}$ & $\begin{array}{c}54 \\
(\mathrm{~S} 2)\end{array}$ & $\begin{array}{c}58 \\
(\mathrm{~S} 2)\end{array}$ & $\begin{array}{c}76 \\
(\mathrm{~S} 1)\end{array}$ & $\begin{array}{l}62 \\
S 2\end{array}$ & $\begin{array}{c}85 \\
(\mathrm{~S} 1)\end{array}$ & $\begin{array}{c}24 \\
(\mathrm{~N} 1)\end{array}$ & $\begin{array}{c}76 \\
(\mathrm{~S} 1)\end{array}$ & $\begin{array}{c}42 \\
(\mathrm{~S} 3)\end{array}$ & $\begin{array}{c}76 \\
(\mathrm{~S} 1)\end{array}$ & $\begin{array}{c}67 \\
(\mathrm{~S} 2)\end{array}$ & $\begin{array}{c}98 \\
(\mathrm{~S} 1)\end{array}$ & $\begin{array}{c}58 \\
(\mathrm{~S} 2)\end{array}$ & $\begin{array}{c}76 \\
\text { (S1) }\end{array}$ \\
\hline 4 & $\begin{array}{c}70 \\
(\mathrm{~S} 2)\end{array}$ & $\begin{array}{c}97 \\
(\mathrm{~S} 1)\end{array}$ & $\begin{array}{c}37 \\
\text { (S3) }\end{array}$ & $\begin{array}{c}62 \\
(\mathrm{~S} 2)\end{array}$ & $\begin{array}{c}66 \\
(\mathrm{~S} 2)\end{array}$ & $\begin{array}{c}90 \\
(\mathrm{~S} 1)\end{array}$ & $\begin{array}{c}70 \\
(\mathrm{~S} 2)\end{array}$ & $\begin{array}{c}98 \\
\text { (S1) }\end{array}$ & $\begin{array}{c}62 \\
(\mathrm{~S} 2)\end{array}$ & $\begin{array}{c}85 \\
(\mathrm{~S} 1)\end{array}$ & $\begin{array}{c}18 \\
\text { (N1) }\end{array}$ & $\begin{array}{c}53 \\
(\mathrm{~S} 2)\end{array}$ & $\begin{array}{c}38 \\
(\mathrm{~S} 3)\end{array}$ & $\begin{array}{c}75 \\
(\mathrm{~S} 1)\end{array}$ & $\begin{array}{c}48 \\
\text { (S3) }\end{array}$ & $\begin{array}{c}84 \\
(\mathrm{~S} 1)\end{array}$ & $\begin{array}{c}18 \\
\text { (N1) }\end{array}$ & $\begin{array}{c}74 \\
(\mathrm{~S} 1)\end{array}$ & $\begin{array}{c}37 \\
\text { (S3) }\end{array}$ & $\begin{array}{c}75 \\
(\mathrm{~S} 1)\end{array}$ & $\begin{array}{c}47 \\
(\mathrm{~S} 3)\end{array}$ & $\begin{array}{c}98 \\
\text { (S1) }\end{array}$ & $\begin{array}{c}37 \\
(\mathrm{~S} 3)\end{array}$ & $\begin{array}{c}75 \\
\text { (S1) }\end{array}$ \\
\hline 5 & $\begin{array}{c}50 \\
(\mathrm{~S} 2)\end{array}$ & $\begin{array}{c}95 \\
\text { (S1) }\end{array}$ & $\begin{array}{c}35 \\
\text { (S3) }\end{array}$ & $\begin{array}{c}61 \\
(\mathrm{~S} 2)\end{array}$ & $\begin{array}{c}52 \\
(\mathrm{~S} 2)\end{array}$ & $\begin{array}{c}87 \\
\text { (S1) }\end{array}$ & $\begin{array}{c}69 \\
(\mathrm{~S} 2)\end{array}$ & $\begin{array}{c}96 \\
\text { (S1) }\end{array}$ & $\begin{array}{c}53 \\
(\mathrm{~S} 2)\end{array}$ & $\begin{array}{c}83 \\
(\mathrm{~S} 1)\end{array}$ & $\begin{array}{c}16 \\
\text { (N1) }\end{array}$ & $\begin{array}{c}46 \\
\text { (S3) }\end{array}$ & $\begin{array}{c}36 \\
\text { (S3) }\end{array}$ & $\begin{array}{c}66 \\
\text { (S2) }\end{array}$ & $\begin{array}{c}36 \\
\text { (S3) }\end{array}$ & $\begin{array}{c}73 \\
\text { (S2) }\end{array}$ & $\begin{array}{c}20 \\
\text { (N1) }\end{array}$ & $\begin{array}{c}67 \\
(\mathrm{~S} 2)\end{array}$ & $\begin{array}{c}34 \\
\text { (S3) }\end{array}$ & $\begin{array}{c}66 \\
\text { (S2) }\end{array}$ & $\begin{array}{c}53 \\
(\mathrm{~S} 2)\end{array}$ & $\begin{array}{c}98 \\
\text { (S1) }\end{array}$ & $\begin{array}{c}36 \\
\text { (S3) }\end{array}$ & $\begin{array}{c}66 \\
\text { (S2) }\end{array}$ \\
\hline 9 & $\begin{array}{c}63 \\
\text { (S2) }\end{array}$ & $\begin{array}{c}97 \\
\text { (S1) }\end{array}$ & $\begin{array}{c}35 \\
\text { (S3) }\end{array}$ & $\begin{array}{c}63 \\
\text { (S2) }\end{array}$ & $\begin{array}{c}67 \\
\text { (S2) }\end{array}$ & $\begin{array}{c}92 \\
\text { (S1) }\end{array}$ & $\begin{array}{c}76 \\
\text { (S1) }\end{array}$ & $\begin{array}{c}98 \\
(\mathrm{~S} 1)\end{array}$ & $\begin{array}{c}55 \\
\text { (S2) }\end{array}$ & $\begin{array}{c}86 \\
\text { (S1) }\end{array}$ & $\begin{array}{c}22 \\
\text { (N1) }\end{array}$ & $\begin{array}{c}55 \\
\text { (S2) }\end{array}$ & $\begin{array}{c}47 \\
\text { (S3) }\end{array}$ & $\begin{array}{l}78 \\
\text { (S1) }\end{array}$ & $\begin{array}{c}46 \\
\text { (S3) }\end{array}$ & $\begin{array}{c}87 \\
\text { (S1) }\end{array}$ & $\begin{array}{c}20 \\
(\mathrm{~N} 1)\end{array}$ & $\begin{array}{l}77 \\
\text { (S1) }\end{array}$ & $\begin{array}{c}35 \\
\text { (S3) }\end{array}$ & $\begin{array}{c}78 \\
(\mathrm{~S} 1)\end{array}$ & $\begin{array}{c}56 \\
(\mathrm{~S} 2)\end{array}$ & $\begin{array}{c}98 \\
\text { (S1) }\end{array}$ & $\begin{array}{c}48 \\
\text { (S3) }\end{array}$ & $\begin{array}{c}78 \\
\text { (S1) }\end{array}$ \\
\hline 10 & $\begin{array}{c}81 \\
(\mathrm{~S} 1)\end{array}$ & $\begin{array}{c}97 \\
(\mathrm{~S} 1)\end{array}$ & $\begin{array}{c}50 \\
(\mathrm{~S} 2)\end{array}$ & $\begin{array}{c}63 \\
(\mathrm{~S} 2)\end{array}$ & $\begin{array}{c}77 \\
(\mathrm{~S} 1)\end{array}$ & $\begin{array}{c}92 \\
(\mathrm{~S} 1)\end{array}$ & $\begin{array}{c}84 \\
(\mathrm{~S} 1)\end{array}$ & $\begin{array}{c}98 \\
(\mathrm{~S} 1)\end{array}$ & $\begin{array}{c}72 \\
(\mathrm{~S} 2)\end{array}$ & $\begin{array}{c}86 \\
(\mathrm{~S} 1)\end{array}$ & $\begin{array}{c}28 \\
(\mathrm{~S} 3)\end{array}$ & $\begin{array}{c}54 \\
(\mathrm{~S} 2)\end{array}$ & $\begin{array}{c}57 \\
(\mathrm{~S} 2)\end{array}$ & $\begin{array}{c}77 \\
\text { (S1) }\end{array}$ & $\begin{array}{c}60 \\
(\mathrm{~S} 2)\end{array}$ & $\begin{array}{c}86 \\
(\mathrm{~S} 1)\end{array}$ & $\begin{array}{c}33 \\
(\mathrm{~S} 3)\end{array}$ & $\begin{array}{c}76 \\
(\mathrm{~S} 1)\end{array}$ & $\begin{array}{c}53 \\
(\mathrm{~S} 2)\end{array}$ & $\begin{array}{c}77 \\
(\mathrm{~S} 1)\end{array}$ & $\begin{array}{c}71 \\
(\mathrm{~S} 2)\end{array}$ & $\begin{array}{c}98 \\
(\mathrm{~S} 1)\end{array}$ & $\begin{array}{c}58 \\
(\mathrm{~S} 2)\end{array}$ & $\begin{array}{c}77 \\
\text { (S1) }\end{array}$ \\
\hline 11 & $\begin{array}{c}49 \\
(\mathrm{~S} 3)\end{array}$ & $\begin{array}{c}97 \\
(\mathrm{~S} 1)\end{array}$ & $\begin{array}{c}35 \\
(\mathrm{~S} 3)\end{array}$ & $\begin{array}{c}62 \\
(\mathrm{~S} 2)\end{array}$ & $\begin{array}{c}53 \\
(\mathrm{~S} 2)\end{array}$ & $\begin{array}{c}92 \\
(\mathrm{~S} 1)\end{array}$ & $\begin{array}{c}71 \\
(\mathrm{~S} 2)\end{array}$ & $\begin{array}{c}98 \\
(\mathrm{~S} 1)\end{array}$ & $\begin{array}{c}53 \\
(\mathrm{~S} 2)\end{array}$ & $\begin{array}{c}86 \\
(\mathrm{~S} 1)\end{array}$ & $\begin{array}{c}19 \\
\text { (N1) }\end{array}$ & $\begin{array}{c}54 \\
(\mathrm{~S} 2)\end{array}$ & $\begin{array}{c}40 \\
(\mathrm{~S} 3)\end{array}$ & $\begin{array}{c}76 \\
(\mathrm{~S} 1)\end{array}$ & $\begin{array}{c}41 \\
\text { (S3) }\end{array}$ & $\begin{array}{c}85 \\
(\mathrm{~S} 1)\end{array}$ & $\begin{array}{c}24 \\
\text { (N1) }\end{array}$ & $\begin{array}{c}75 \\
(\mathrm{~S} 1)\end{array}$ & $\begin{array}{c}40 \\
(\mathrm{~S} 3)\end{array}$ & $\begin{array}{c}76 \\
(\mathrm{~S} 1)\end{array}$ & $\begin{array}{c}51 \\
(\mathrm{~S} 2)\end{array}$ & $\begin{array}{c}98 \\
\text { (S1) }\end{array}$ & $\begin{array}{c}40 \\
(\mathrm{~S} 3)\end{array}$ & $\begin{array}{c}76 \\
\text { (S1) }\end{array}$ \\
\hline 12 & $\begin{array}{c}44 \\
\text { (S3) }\end{array}$ & $\begin{array}{c}98 \\
(\mathrm{~S} 1)\end{array}$ & $\begin{array}{c}26 \\
\text { (S3) }\end{array}$ & $\begin{array}{c}63 \\
\text { (S2) }\end{array}$ & $\begin{array}{c}45 \\
(\mathrm{~S} 3)\end{array}$ & $\begin{array}{c}93 \\
(\mathrm{~S} 1)\end{array}$ & $\begin{array}{c}63 \\
(\mathrm{~S} 2)\end{array}$ & $\begin{array}{c}99 \\
\text { (S1) }\end{array}$ & $\begin{array}{c}61 \\
(\mathrm{~S} 2)\end{array}$ & $\begin{array}{c}87 \\
(\mathrm{~S} 1)\end{array}$ & $\begin{array}{c}17 \\
\text { (N1) }\end{array}$ & $\begin{array}{c}55 \\
(\mathrm{~S} 2)\end{array}$ & $\begin{array}{c}38 \\
\text { (S3) }\end{array}$ & $\begin{array}{c}78 \\
(\mathrm{~S} 1)\end{array}$ & $\begin{array}{c}37 \\
\text { (S3) }\end{array}$ & $\begin{array}{c}88 \\
\text { (S1) }\end{array}$ & $\begin{array}{c}24 \\
\text { (N1) }\end{array}$ & $\begin{array}{c}87 \\
(\mathrm{~S} 1)\end{array}$ & $\begin{array}{c}44 \\
(\mathrm{~S} 3)\end{array}$ & $\begin{array}{c}78 \\
(\mathrm{~S} 1)\end{array}$ & $\begin{array}{c}46 \\
(\mathrm{~S} 3)\end{array}$ & $\begin{array}{c}98 \\
\text { (S1) }\end{array}$ & $\begin{array}{c}38 \\
\text { (S3) }\end{array}$ & $\begin{array}{c}78 \\
\text { (S1) }\end{array}$ \\
\hline 13 & $\begin{array}{c}81 \\
(\mathrm{~S} 1)\end{array}$ & $\begin{array}{c}98 \\
(\mathrm{~S} 1)\end{array}$ & $\begin{array}{c}48 \\
\text { (S3) }\end{array}$ & $\begin{array}{c}63 \\
(\mathrm{~S} 2)\end{array}$ & $\begin{array}{c}77 \\
\text { (S1) }\end{array}$ & $\begin{array}{c}93 \\
(\mathrm{~S} 1)\end{array}$ & $\begin{array}{c}80 \\
\text { (S1) }\end{array}$ & $\begin{array}{c}98 \\
(\mathrm{~S} 1)\end{array}$ & $\begin{array}{c}70 \\
(\mathrm{~S} 2)\end{array}$ & $\begin{array}{c}87 \\
(\mathrm{~S} 1)\end{array}$ & $\begin{array}{c}28 \\
(\mathrm{~S} 3)\end{array}$ & $\begin{array}{c}56 \\
(\mathrm{~S} 2)\end{array}$ & $\begin{array}{c}57 \\
(\mathrm{~S} 2)\end{array}$ & $\begin{array}{c}79 \\
(\mathrm{~S} 1)\end{array}$ & $\begin{array}{c}59 \\
(\mathrm{~S} 2)\end{array}$ & $\begin{array}{c}88 \\
(\mathrm{~S} 1)\end{array}$ & $\begin{array}{c}31 \\
(\mathrm{~S} 3)\end{array}$ & $\begin{array}{c}87 \\
(\mathrm{~S} 1)\end{array}$ & $\begin{array}{c}49 \\
(\mathrm{~S} 3)\end{array}$ & $\begin{array}{c}79 \\
(\mathrm{~S} 1)\end{array}$ & $\begin{array}{c}66 \\
(\mathrm{~S} 2)\end{array}$ & $\begin{array}{c}98 \\
(\mathrm{~S} 1)\end{array}$ & $\begin{array}{c}58 \\
(\mathrm{~S} 2)\end{array}$ & $\begin{array}{c}79 \\
\text { (S1) }\end{array}$ \\
\hline 20 & $\begin{array}{c}83 \\
(\mathrm{~S} 1) \\
\end{array}$ & $\begin{array}{c}97 \\
(\mathrm{~S} 1) \\
\end{array}$ & $\begin{array}{r}51 \\
(\mathrm{~S} 2) \\
\end{array}$ & $\begin{array}{c}63 \\
(\mathrm{~S} 2) \\
\end{array}$ & $\begin{array}{c}77 \\
(\mathrm{~S} 1) \\
\end{array}$ & $\begin{array}{c}92 \\
(\mathrm{~S} 1) \\
\end{array}$ & $\begin{array}{c}84 \\
(\mathrm{~S} 1) \\
\end{array}$ & $\begin{array}{c}98 \\
(\mathrm{~S} 1) \\
\end{array}$ & $\begin{array}{r}73 \\
(\mathrm{~S} 2) \\
\end{array}$ & $\begin{array}{c}86 \\
(\mathrm{~S} 1) \\
\end{array}$ & $\begin{array}{c}30 \\
\text { (S3) } \\
\end{array}$ & $\begin{array}{r}54 \\
(\mathrm{~S} 2) \\
\end{array}$ & $\begin{array}{r}58 \\
(\mathrm{~S} 2) \\
\end{array}$ & $\begin{array}{c}77 \\
\text { (S1) } \\
\end{array}$ & $\begin{array}{r}64 \\
(\mathrm{~S} 2) \\
\end{array}$ & $\begin{array}{c}86 \\
(\mathrm{~S} 1) \\
\end{array}$ & $\begin{array}{c}33 \\
(\mathrm{~S} 3) \\
\end{array}$ & $\begin{array}{c}76 \\
(\mathrm{~S} 1) \\
\end{array}$ & $\begin{array}{r}54 \\
(\mathrm{~S} 2) \\
\end{array}$ & $\begin{array}{c}77 \\
(\mathrm{~S} 1) \\
\end{array}$ & $\begin{array}{c}72 \\
\text { (S2) } \\
\end{array}$ & $\begin{array}{c}98 \\
(\mathrm{~S} 1) \\
\end{array}$ & $\begin{array}{r}58 \\
(\mathrm{~S} 2) \\
\end{array}$ & $\begin{array}{c}77 \\
\text { (S1) } \\
\end{array}$ \\
\hline $\mathrm{S} 2=\mathrm{N}$ & deratel & y suita & & & IV. & na & dita & & & $=$ & er & & & & & & & & & & & & & \\
\hline
\end{tabular}


Table 6. Cont.

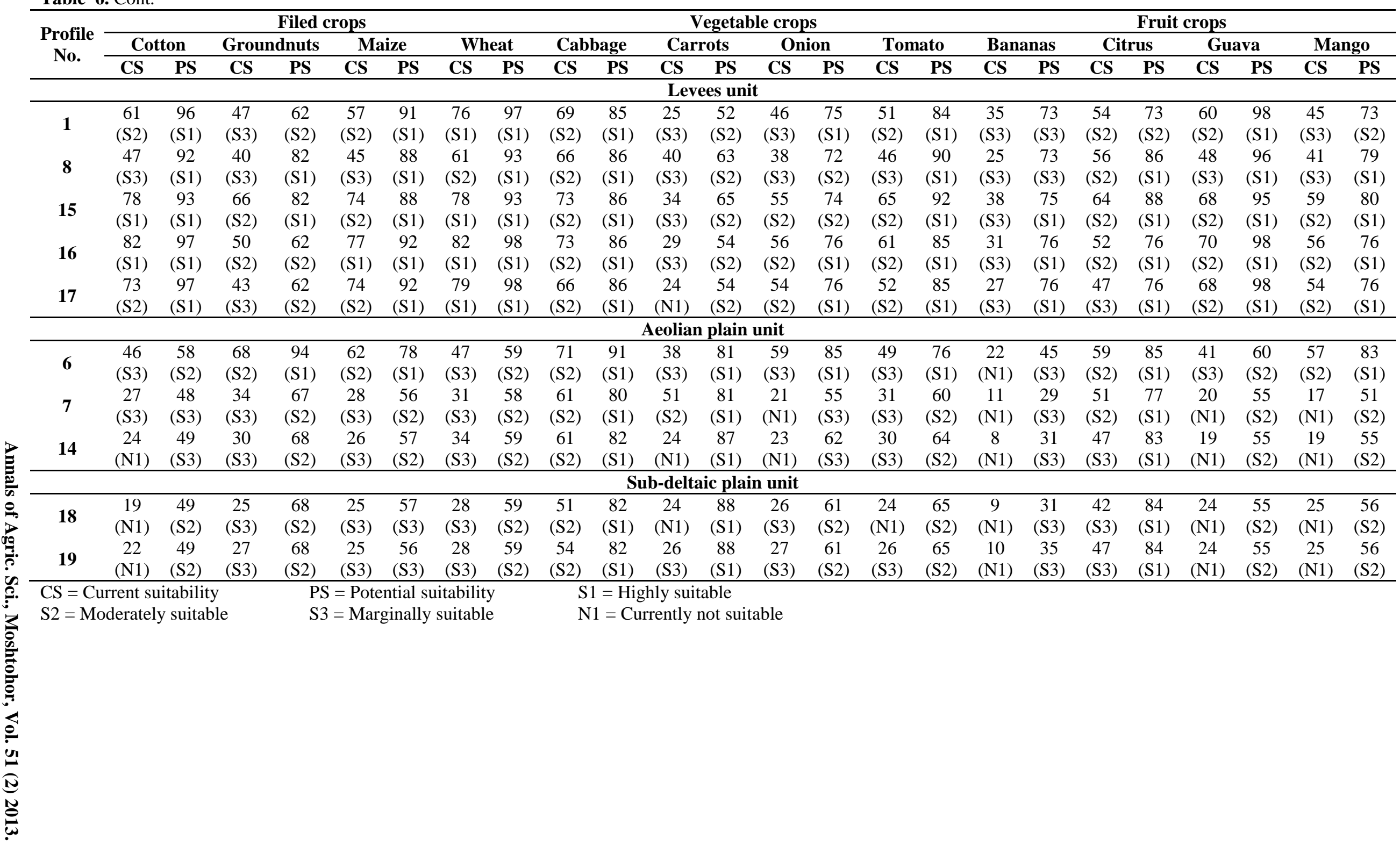


Table 7. Distribution of suitability of potential suitability classes for the selected crops

\begin{tabular}{|c|c|c|c|c|c|c|c|c|c|c|c|c|}
\hline \multirow{3}{*}{ Crop } & \multicolumn{12}{|c|}{ Geomorphic units } \\
\hline & \multicolumn{3}{|c|}{ Alluvial plain } & \multicolumn{3}{|c|}{ Levees } & \multicolumn{3}{|c|}{ Aeolian plain } & \multicolumn{3}{|c|}{ Sub-Deltaic plain } \\
\hline & S1 & S2 & S3 & S1 & $\mathbf{S 2}$ & S3 & S1 & $\mathbf{S 2}$ & S3 & S1 & $\mathbf{S 2}$ & S3 \\
\hline Cotton & $100 \%$ & ---- & ---- & $100 \%$ & ---- & ---- & ---- & 33.3 & 66.7 & ---- & $100 \%$ & ---- \\
\hline Groundnuts & ---- & $100 \%$ & & $40 \%$ & $60 \%$ & ---- & 33.3 & 66.7 & ---- & ---- & $100 \%$ & ---- \\
\hline Maize & $100 \%$ & ---- & ---- & $100 \%$ & ---- & ---- & 33.3 & 66.7 & ---- & ---- & ---- & $100 \%$ \\
\hline Sesame & ---- & $100 \%$ & ---- & $40 \%$ & $60 \%$ & ---- & ---- & ---- & ---- & $100 \%$ & ---- & ---- \\
\hline Soya & ---- & $90 \%$ & $10 \%$ & & $80 \%$ & $20 \%$ & ---- & ---- & $100 \%$ & ---- & ---- & $100 \%$ \\
\hline Sunflower & $100 \%$ & ---- & ---- & $100 \%$ & ---- & ---- & 33.3 & 66.7 & ---- & ---- & $100 \%$ & ---- \\
\hline Wheat & $100 \%$ & ---- & ---- & $100 \%$ & ---- & ---- & ---- & $100 \%$ & ---- & ---- & $100 \%$ & ---- \\
\hline Cabbage & $100 \%$ & ---- & ---- & $100 \%$ & ---- & ---- & $100 \%$ & ---- & --- & $100 \%$ & ---- & ---- \\
\hline Carrots & ---- & $90 \%$ & $10 \%$ & ---- & $100 \%$ & ---- & $100 \%$ & ---- & ---- & $100 \%$ & ---- & ---- \\
\hline Green pepper & ---- & $90 \%$ & $10 \%$ & ---- & $80 \%$ & $20 \%$ & 33.3 & 66.7 & ---- & ---- & $100 \%$ & ---- \\
\hline Onion & $80 \%$ & $20 \%$ & ---- & $80 \%$ & $20 \%$ & ---- & 33.3 & 66.7 & ---- & ---- & $100 \%$ & ---- \\
\hline Pea & $100 \%$ & ---- & ---- & $100 \%$ & ---- & ---- & 33.3 & 66.7 & ---- & ---- & $100 \%$ & ---- \\
\hline Potato & $100 \%$ & ---- & ---- & $100 \%$ & ---- & ---- & 33.3 & 66.7 & ---- & ---- & $100 \%$ & ---- \\
\hline Tomato & $90 \%$ & $10 \%$ & ---- & $100 \%$ & ---- & ---- & 33.3 & 66.7 & ---- & ---- & $100 \%$ & ---- \\
\hline Banana & $70 \%$ & $30 \%$ & ---- & $60 \%$ & $40 \%$ & ---- & ---- & ---- & $100 \%$ & ---- & ---- & $100 \%$ \\
\hline Citrus & $90 \%$ & $10 \%$ & ---- & $80 \%$ & $20 \%$ & ---- & $100 \%$ & ---- & ---- & $100 \%$ & ---- & ---- \\
\hline Guava & $100 \%$ & ---- & ---- & $100 \%$ & ---- & ---- & ---- & $100 \%$ & ---- & ---- & $100 \%$ & ---- \\
\hline Mango & $90 \%$ & $10 \%$ & ---- & $80 \%$ & $20 \%$ & ---- & 33.3 & 66.7 & ---- & ---- & $100 \%$ & ---- \\
\hline
\end{tabular}




\section{References}

Abd El-Salam, M.A. 2001. Studies on potassium in some soils of Qalubiya, Egypt. M.Sc. Thesis, Fac. of Agric., Moshtohor, Zagazig Univ., Benha branch, Egypt.

Bassuony, M.A. 2012. A Study on irrigation water quality in some regions of Qalubiya Governorate. M.Sc. Thesis, Fac. of Agric., Benha Univ., Egypt.

Bot, A. and Benites, J. 2005. The importance of soil organic matter. Key to drought-resistant soil and sustained food and production. FAO, Rome.

Brewer, R. 1964. Fabric and mineral analysis. John Wiley \& Sons, New York, USA.

Buck, B. J. and Van Hoesen, J. G. 2002. Snowball morphology and SEM analysis of pedogenic gypsum, Southern New Mexico, U.S.A. J. Arid Environ., 51: 469-487.

El-Shanawany, E.A. 1992. Pedological studies of some zone of encroachment between the Nile Delta and the desert in Qalubiya Governorate. Ph.D. Thesis, Fac. of Agric., Zagazig Univ., Egypt.

FAO. 1976. A framework for land evaluation. Soil Bull. No. 32, Rome, Italy.

FAO. 2006. Guidelines for soil profile description, FAO, ISRIC, Publication, Rome, Italy.

Hashemi, S.S., Baghernejad, M. and Khademi, H. 2011. Micromorphology of gypsum crystals in Southern Iranian soils under different moisture regimes. J. Agr. Sci. Tech., 13: 273-288

Mishra, A. 2007. Land suitability classification for different crops. Orissa Review. November, 2007

Munsell soil color charts 1971. Munsell color company, Inc., Baltimore, Maryland, 21218, U.S.A

Rice, C.W. 2002. Organic matter and nutrient dynamics. In: Encyclopedia of Soil Science: 925928. New York, USA, Marcel Dekker Inc.

Ritung, S.; Wahyunto, A. and Hidayat, H. 2007. Land suitability evaluation with a case map of Aceh Barat District. Indonesian Soil Research Institute and World Agroforestry Centre, Bogor, Indonesia.
Ryan, J., Estefan, G. and Rashid, A. 2003. Soil and plant analysis laboratory manual. $2^{\text {nd }}$ edition. ICARDA, Aleppo, Syria.

Samy, Y. and Metwally, H.I. 2012. Gypsum crystal habitat as an evidence for aridity and stagnation, Northeast of the Nile Delta coast, Egypt. Australian J. of Basic and Applied Sci., 6(6): 442-450.

Shalaby, A. and Gad, A. 2010. Urban sprawl impact assessment on the fertile agricultural land of Egypt using remote sensing and digital soil database, Case study: Qalubiya Governorate. USEgypt Workshop on Space Technology and Geoinformation for Sustainable Development, Cairo, Egypt 14-17 June.

Singh Alka, A.K.; Vasisht, R.K. and Das, D.K. 2008. Adoption of integrated pest management practices in paddy and cotton: A Case study in Haryana and Punjab, Agricultural Economics Res. Rev. 21: 221-226.

Soil Survey Staff. 1993. Soil Survey Manual Handbook 18, US. Gov. Print. off., Washington, DC.

Sys, C. 1985. Land characteristics and utilities and methods of irrigation them. FAO, Soils Bull. No. 55 , Rome, Italy.

Sys, C. and Verheye, W. 1978. An attempt to the evaluation of physical land characteristics for irrigation according to the FAO Framework for Land Evaluation. Int. Train. Center for Post Grad. Soil Sci., Ghent, Belgium.

Sys, C., Van-Ranst, E. and Debaveye, J. 1991. Land evaluation. Part I and II, Lecture Notes. Ghent Univ., Belgium.

Sys, C., Van-Ranst, E., Debaveye, J. and Beernaert, F. 1993. Land evaluation. Part III Crop requirements, Lecture Notes., Ghent Univ., Belgium.

USDA. 2009. Soil survey field and laboratory methods manual. Soil Survey Investigations Report No. 51, Version 1.0. R. Burt (ed.). U.S. Department of Agriculture, Natural Resources Conservation Service. 


\section{تصنيف صلاحية بعض أراضي القليويبة للزراعة}

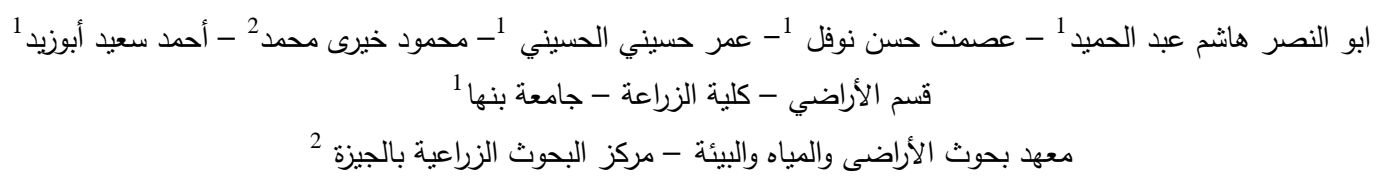

\section{الملخص العربي}

يهدف هذا البحث إلى تقييم صداحية بعض أراضي محافظة القليوبية للزراعة والمروية ولزراعة محاصيل معينة. تبلغ المساحة المنزرعة

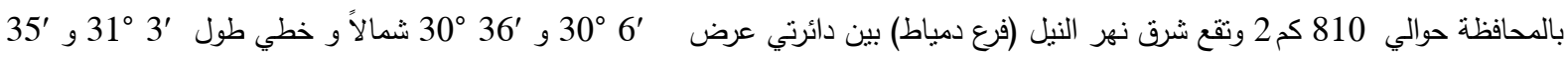

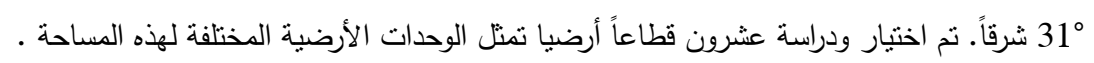

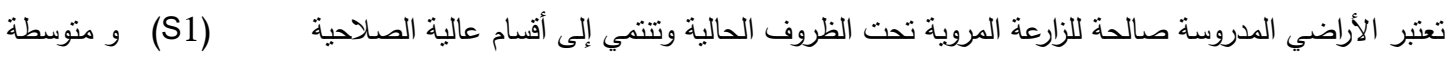
الصلاحية (S2) و هامشية الصلاحية (S3) يعتبر قوام التربة والملوحة والقلوية أهم محددات (معوقات) الزراعة تحت الظروف الحالية، بينما تحت

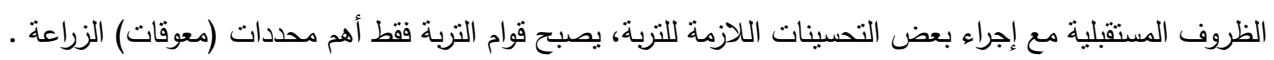

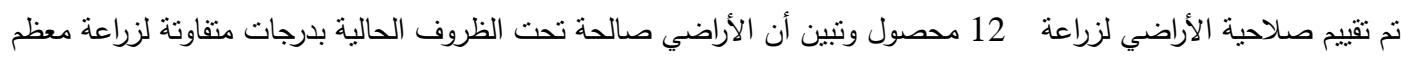

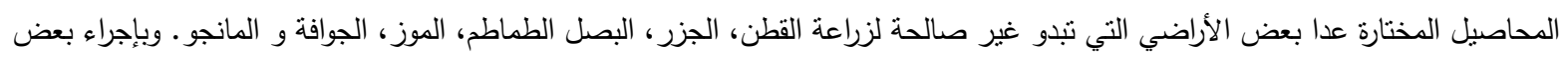
التحسينات اللازمة لهذه الأراضي تصبح صالحة لزراعة كافة المحاصيل المختارة. 